\title{
AN EXPERIMENT FOR OBSERVING VHE GAMMA RAY SOURCES
}

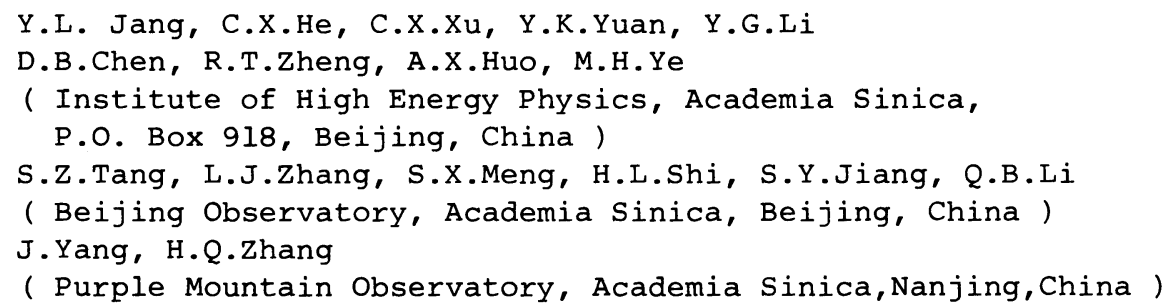

ABSTRACT. An apparatus design is described. It is for detecting VHE gamma ray point sources by means of the atmospheric Cerenkov technique. Obviously, the improvement of flux sensitivity and discrimination between gamma ray and isotropic proton showers is still a key problem. Of course, it is necessary to set up more observatories and to track an object continuously with several facilities. With this in mind, we decided to develop an experiment for observing VHE gamma ray sources in China. As a first step, we will set up an apparatus which consists of three $1.5 \mathrm{~m}$ diameter searchlight morrors at Xinglong station of Beijing Observatory, Xinglong county, Hebei province $\left(40^{\circ}\right.$. $4 \mathrm{~N}, 117^{\circ} .5 \mathrm{E}$, altitude $940 \mathrm{~m}$ ). The observation will start in 1988. Then, the second apparatus will be set up at Delingha station of Purple Mountain Observatory, Delingha county, Qinghai porvince $\left(37^{\circ} .22 \mathrm{~N}\right.$, altitude $\left.3204 \mathrm{~m}\right)$. Both the sites are far from air and light pollution, and have suitable meteological condition for Cerenkov light detection as well as quite convenient facilities for transportation. Some probable technical improvements are also discussed in this paper. 